The motion of the gas stream and the apparent position of the X-ray source strongly suggest that the X-rays are being produced through the interaction of the gas stream and the unseen companion. The high energies of the X-rays imply that large accelerations are involved-accelerations such as might be produced by the intense gravitational field of a collapsed object. The lower limits placed on the secondary mass are too high for a white dwarf and probably rule out a neutron star. The lack of a supernova remnant also argues against the secondary being a neutron star. This raises the distinct possibility that the secondary is a black hole. Further observations around secondary eclipse could test this possibility.

The good agreement between the velocity variations of $\mathrm{HDE}$ 226868 and the X-ray variations of Cyg X-1, combined with their close positional coincidence, argues strongly for their identity. Furthermore, I have found $\lambda 4686$ of HeII in emission on plates taken by Humphreys, and this and other emission lines may be marginally visible on my plates. This would be very unusual for a BOIb star, but $\lambda 4686$ has been seen in emission in the spectra of Sco X-1 (ref. 8) and Cyg X-2 (ref. 9). Thus the identity of Cyg X-1 and HDE 226868 appears almost certain.

Several problems remain, however. I have already discussed the problems involved in deriving a period for the X-ray variations. The observations by Matteson ${ }^{5}$ suggest that an additional complication may be present. He finds that Cyg $\mathrm{X}-1$ varies by a factor of seven while the observations summarized by Dolan ${ }^{2,4}$ indicate variations of no more than a factor of two. The difference here may be due to some of the experimental problems discussed by Dolan ${ }^{2}$. The difference might also be caused by variations in the synchrotron component of the source. If this were the case, it would be quite easy to derive a spurious period for the X-ray variations from the limited data that are currently available. The true nature of the X-ray variation can only be adequately determined by continuous monitoring of Cyg X-1 over a large energy range. Ideally this should be coordinated with optical and radio observations.

This work was supported through grants from the National Research Council of Canada.

\section{T. Bolton}

\section{David Dunlap Observatory,}

University of Toronto,

Richmond Hill, Ontario

Received January 3, 1972.

1 Braes, L. L. E., and Miley, G. K., Nature, 232, 246 (1971).

2 Dolan, J. F., Space Sci. Rev., 10, 830 (1969).

3 Plavec, M. Pub. Astron. Soc. Pacific, 82, 957 (1970).

${ }^{4}$ Dolan, J. F., Nature, 233, 109 (1971).

5 Matteson, J. L., Bull. Amer. Astron. Soc., 3, 456 (1971).

6 Brini, D., Ciriegi, U., Fuligni, F., Moretti, E., and Vespignani, G., Astrophys. J., 149, 429 (1967).

7 Agrawal, P. C., Gokhale, G. S., Iyengar, V. S., Kunte, P. K. Manchanda, R. K., and Sreekantan, B. V., Nature, 232, 38 (1971).

${ }^{8}$ Hiltner, W. A., and Mook, D., Ann. Rev. Astron. Astrophys., 8, $139(1970)$.

${ }^{9}$ Kraft, R. P., and Demoulin, M., Astrophys. J. Lett., 150, L83 (1967).

\section{Persei an X-ray Source?}

WE suggest that the peculiar star $X$ Per is connected with the Uhuru X-ray source 2ASE $0352+30$.

The sixth magnitude irregular variable X Per (BS 1209= HD 24534), situated in the Per OB2 (II Per) association at $\alpha=3$ h $52.3 \mathrm{~min}, \delta=30^{\circ} 54^{\prime}(1950)$, is of spectral type Opev and shows a large uitraviolet excess ${ }^{1,2}$. Blaauw ${ }^{3}$ has suggested that it is the remnant of a supernova explosion that gave rise to the runaway star $\xi$ Per. In a subsequent discussion on the relationship between groupings of early-type stars and X-ray sources, Braes and Hovenier ${ }^{4}$ predicted that X-ray emission might be observable from the region of Per OB2. Such an $\mathrm{X}$-ray source, 2ASE $0352+30$, has now been detected $(R$. Giacconi et al., unpublished catalogue of Uhuru X-ray sources). The quoted Uhuru position of this source, which has a $2 \sigma$ uncertainty of $5^{\prime} \times 15^{\prime}$, agrees to within $2^{\prime}$ with that of $X$ Per. These arguments lead us to believe that the X-ray source is associated with this star.

Because some other X-ray sources are known to be radio emitters, we have searched for $1,415 \mathrm{MHz}$ radiation from the $\mathrm{X}$ Per region with the Westerbork synthesis radio telescope. Twelve-hour observations made on both May 24 and 27, 1971, failed to detect any source brighter than 0.005 flux units within the 2ASE $0352+30$ error box. Because of the extreme radio variability of $\mathrm{X}$-ray sources, however, these measurements are not conclusive. Continued monitoring of $X$ Per at both optical and radio wavelengths is needed.

We thank the ASE group for providing us with data in advance of their publication. The Westerbork Radio Observatory is operated by the Netherlands Foundation for Radio Astronomy with the financial support of the Netherlands Organization for the Advancement of Pure Research (ZWO).

L. L. E. BRaEs

G. K. MILEY

Leiden Observatory,

Leiden 2401,

The Netherlands

Received December 21, 1971.

1 Borgman, J., and Blaauw, A., Bull. Astron. Insts. Netherlands, 17, 358 (1964).

2 Kukarkin, B. V., Kholopov, P. N., Efremov, Yu. N., Kukarkina, N. P., Kurochkin, N. E., Medvedeva, G. I., Perova, N. B., Fedorovich, V. P., and Frolov, M. S., General Catalogue of Variable Stars, 2, third edition (Moscow, 1970).

3 Blaauw, A., Bull. Astron. Insts. Netherlands, 15, 265 (1961).

${ }^{4}$ Braes, L. L. E., and Hovenier, J. W., Nature, 209, 360 (1966).

\section{Possible Identification of X Persei with an X-ray Source}

COMPARISON of the Yale catalogue ${ }^{1}$ with a recent catalogue of 116 X-ray sources observed with the Uhuru satellite (private communication from R. Giacconi, H. Gursky, E. Kellogg, S. Murray, E. Schreier and H. Tananbaum) shows that the position of the irregular variable star X Persei (see Table 1) coincides with that of the X-ray source 2 ASE $0352+30$. The total number of chance coincidences of bright stars to within 0.5 arc min in $\alpha$ and $0^{\circ} .1$ in $\delta$ of the Uhuru X-ray source positions is estimated to be $\sim 1$-the exact number of chance coincidences is difficult to evaluate because both the bright stars and the X-ray sources have a non-random distribution on the sky - but the very unusual nature of X Persei lends some support to the speculation that the positional agreement of this star with an X-ray source may not be a chance coincidence. The spectrum of $\mathrm{X}$ Persei is classified as $\mathrm{O}$ pe, and is peculiar because it is strongly veiled.

Table 1 Comparisons of the Positions of $X$ Persei and 2ASE $0352+30$

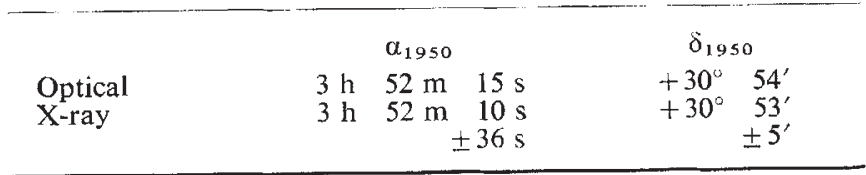

Quoted errors are $90 \%$ confidence limits.

In 1961 Blaauw $^{2}$ suggested that $X$ Persei and the highvelocity $O$ star $\xi$ Persei originally formed a physical binary system which disintegrated when X Persei became a supernova. 